\title{
A polytrauma patient with an unusual posterior fracture-dislocation of the femoral head: a case report
}

\author{
Juan Rodriguez-Martin · Juan Pretell-Mazzini • \\ Miguel Angel Porras-Moreno • \\ Yolanda Hernanz-Gonzalez • Carlos Resines-Erasun
}

Received: 8 July 2008/Accepted: 20 December 2009/Published online: 12 January 2010

(C) Springer-Verlag 2010

\begin{abstract}
We report a case of a 27 -year-old man who was involved in a high-speed car accident. He sustained multiple organ damage including multiple brain petechiae suggesting diffuse axonal damage, aortic dissection, retroperitoneal haematoma and a fracture-dislocation of the right hip with a femoral head fracture and an ipsilateral intertrochanteric fracture. Due to the general condition of the patient, physiological stabilisation was prioritized, and at 2 weeks the fracture-dislocation of the hip was treated with a proximal femoral nail for the intertrochanteric fracture and Herbert screws for the femoral head fracture. Postoperatively, two episodes of recurrent hip dislocation occurred, and this was stabilized eventually with a Steinman pin inserted across the hip joint and taken out 1 month later. Weight-bearing was allowed according to clinical and radiographical assessments. Heterotopic ossification developed around the hip joint, but without evidence of AVN or osteoarthritis. At 18-months follow-up, the fractures had healed and the patient had a Harris Hip score of 79.1. Anatomical reduction and stable fixation of fracturedislocations of the hip are important for achieving an acceptable result.
\end{abstract}

\footnotetext{
J. Rodriguez-Martin ( $(\square)$

Infanta Leonor Hospital,

Gran Via del Este, 60, 28032 Madrid, Spain

e-mail: juanrodmar@yahoo.es

J. Pretell-Mazzini - M. A. Porras-Moreno .

Y. Hernanz-Gonzalez · C. Resines-Erasun

Orthopedic Surgery, Hospital Doce de Octubre,

Av. de Córdoba s/n N-IV Km 5, 4, 28041 Madrid, Spain
}

Keywords Hip dislocation - Femoral head fracture Heterotopic ossification · Prognosis

\section{Introduction}

A fracture of the femoral head with hip dislocation is a rare and severe injury [1-3]. This was initially considered an industrial injury but the advent of high-speed travel has contributed to an increased incidence [1, 4]; this is reported to vary from 6 to $16 \%$ of all hip dislocations [2-6]. The first report of this injury was in 1869 from an autopsy by Birkett of a victim of a fall. In 1957, Pipkin reviewed a series of 25 cases and proposed a classification of femoral head fractures associated with posterior dislocation of the hip $[4,7]$. The mechanism of the injury in the majority of cases is a dashboard injury in a seat occupant unprotected by a seat belt $[2-4,7,8]$. It is caused by a force applied parallel to the femur in a proximal direction with the hip in varying amounts of flexion; if the hip is flexed less than $60^{\circ}$, the femoral head impinges against the firm posterior superior rim and a fracture of the femoral head may occur $[4,7]$. Direct trauma to the greater trochanter has also been described as a mechanism [4]. Because of the rarity of this injury, many series in the literature are with short follow-ups or small numbers of patients and, in our case, the presence of an intertrochanteric fracture is an even more unusual combination which, to the best of our knowledge, has been described previously in 3 other cases [1, 5, 9]. Accurate diagnosis of this complex injury is often difficult, as is its treatment which is based on anatomical reduction and restoration of hip stability by removal of the interposed bone fragments and rigid fixation of the femoral head fracture $[6,10]$. We describe a femoral head 


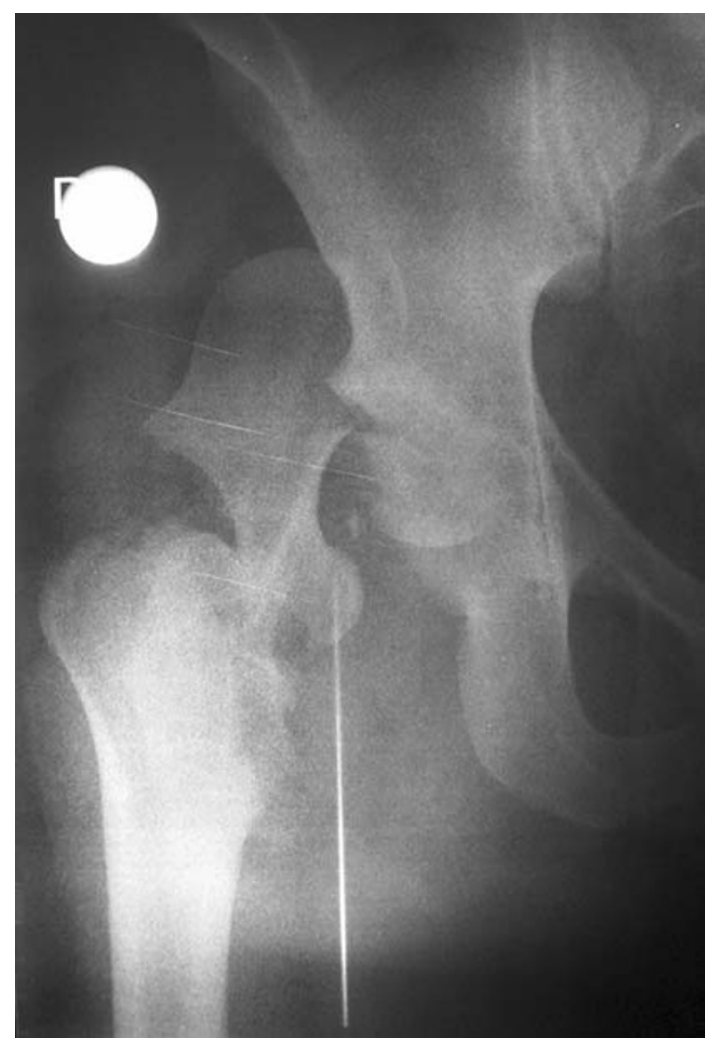

Fig. 1 Initial right hip x-ray showing a posterior fracture-dislocation of the femoral head with an associated intertrochanteric fracture fracture associated with an intertrochanteric fracture and hip dislocation and the general approach to this rare entity.

\section{Case report}

A 27-year-old man was injured in a high-speed car accident. The patient was transferred by helicopter to our polytrauma unit where, on arrival, he had a Glasgow score of six and haemodynamic instability. There were multiple lacerations with signs of thoracic, abdominal, cranial and limb trauma. X-rays of the chest and pelvis were performed showing bilateral clavicle fractures, a widened mediastinum and a fracture-dislocation of the right hip (Fig. 1). A computed tomography (CT) body scan was obtained and showed multiple petechiae in the brain suggesting diffuse axonal injury, a haemorrhagic left frontal basal contusion and a small subarachnoid haemorrhage; a mediastinal haematoma with aortic dissection, left pneumothorax and right lung contusion; retroperitoneal haematoma with active bleeding from the left suprarenal artery and superior mesenteric artery; and a complex fracture-dislocation of the right hip (Fig. 2a-d). The initial management included intracranial pressure monitoring and conservative treatment of the abdominal injuries. The aortic dissection
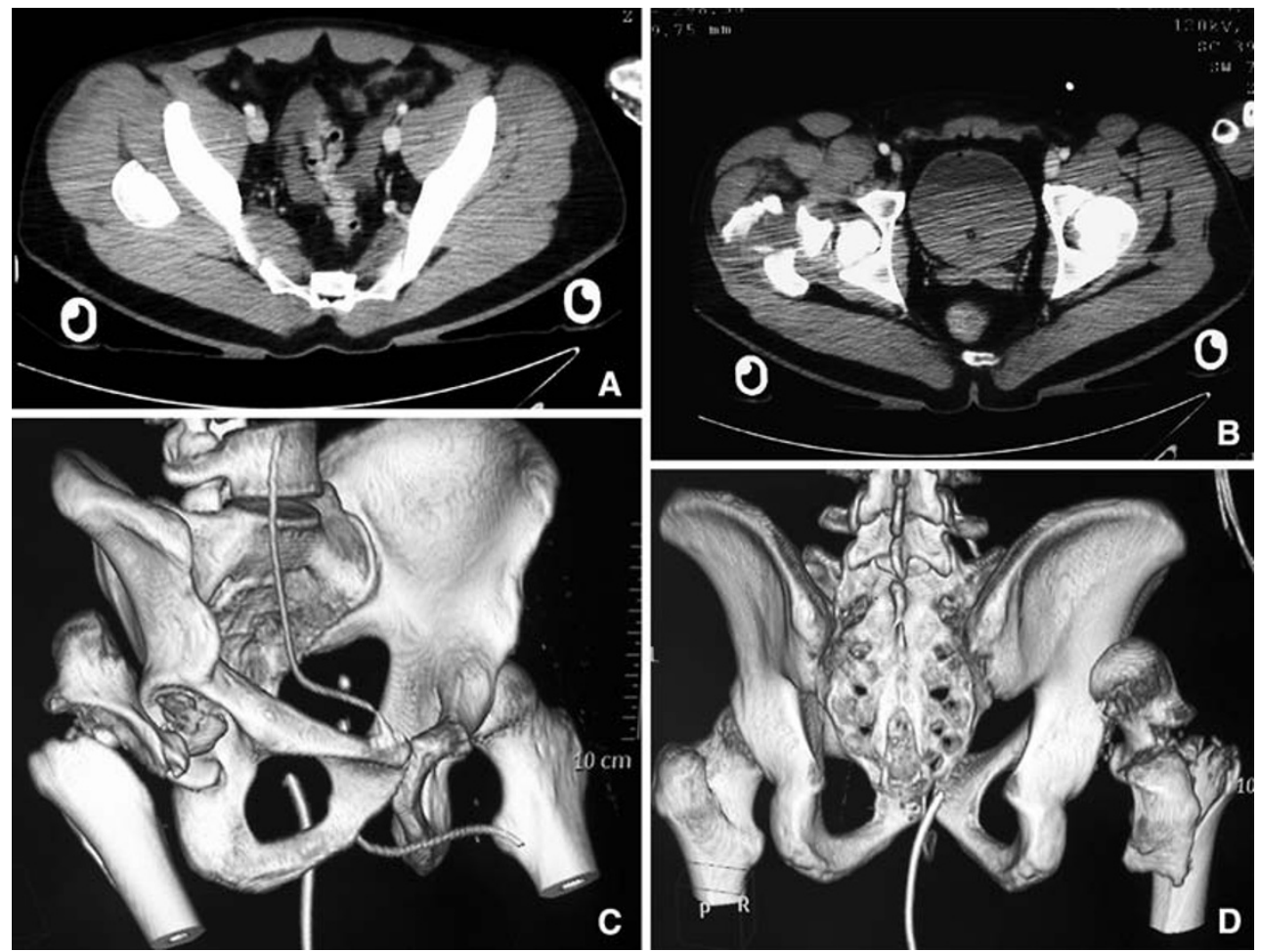

Fig. 2 CT scan of the pelvis. a Coronal view. Posterior fracture-dislocation of the femoral head. b Coronal view. Intertrochanteric hip fracture. c 3D reconstruction image. Anterior view. d 3D reconstruction image. Posterior view 
was repaired with a vascular prosthesis (Hemashield Platinum Woven Double Velour Vascular Graft, Boston Scientific, NJ, USA) (Fig. 3). Supracondylar skeletal traction was applied as initial management of the hip injury whilst the patient received intensive supportive care. The patient subsequently developed multiorgan failure which prevented surgical intervention to the hip until 15 days after the injury. At surgery, the patient was placed on an

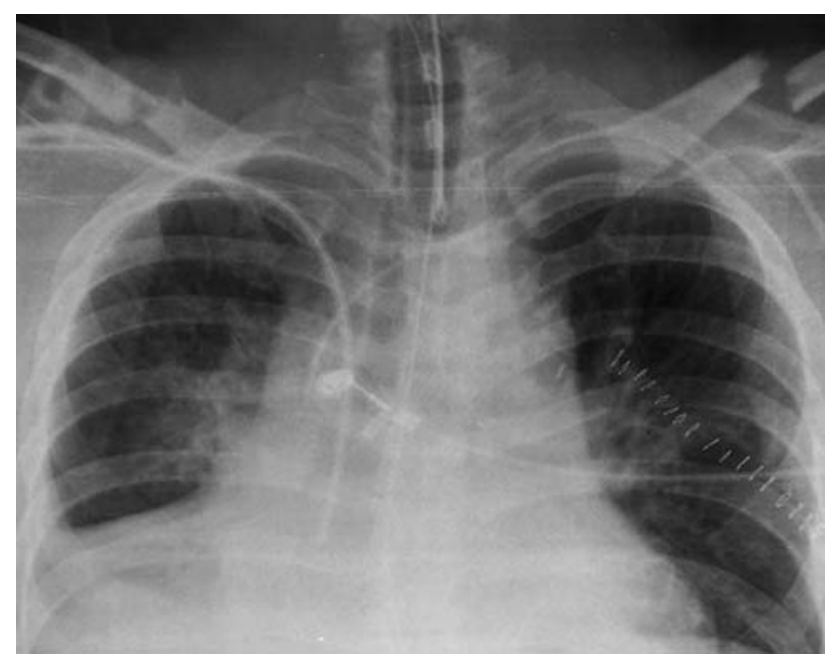

Fig. 3 Postoperative x-ray. Left thoracotomy was performed to repair the aortic dissection. Bilateral clavicle fracture

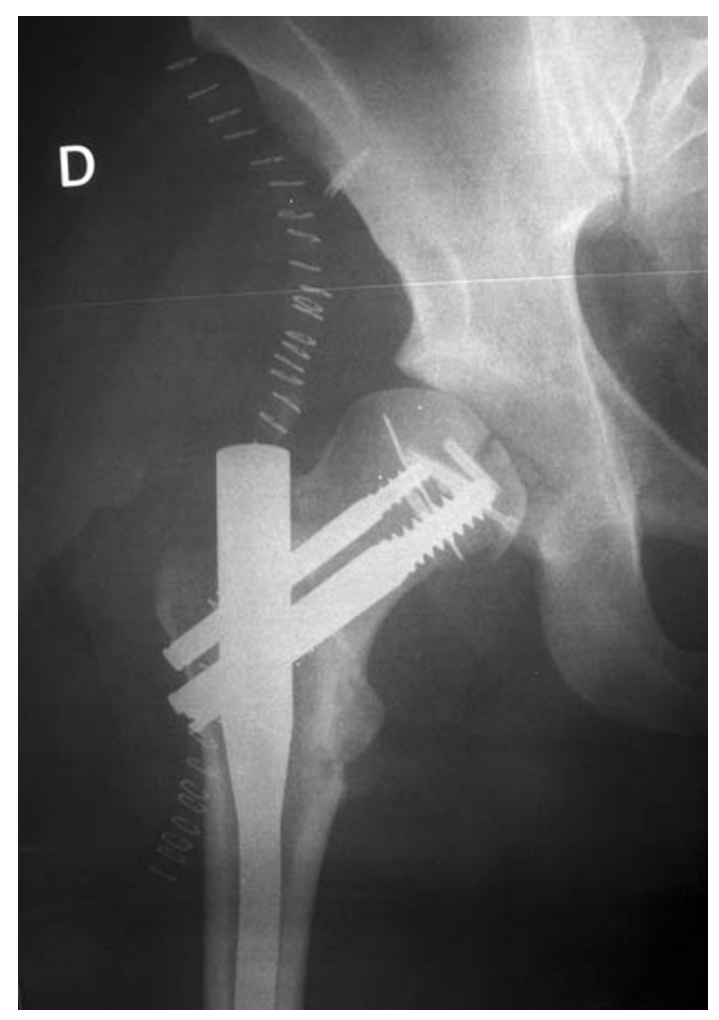

Fig. 4 Postoperative hip x-ray. Anteroposterior view orthopaedic fracture table and an anterior approach to the hip performed. The fracture fragments of the femoral head were reduced and fixed with four Herbert screws (Zimmer Inc., Warsaw, IN). A cephalomedullary Proximal Femoral Nail (Synthes, Solothurn, Switzerland) was used to stabilize the intertrochanteric fracture. An extension of the anterior approach was allowed insertion of the femoral nail. A 5-mm suture anchor (Twinfix, Smith and Nephew, Andover, MA, USA) was used for reattachment of the rectus femoris muscle onto the anterior inferior iliac spine (Fig. 4). The patient was transferred back to the intensive care unit and remained for a further 25 days. Nine days after surgery, the x-ray control images revealed a recurrent posterior dislocation of the hip. Closed reduction was performed in the intensive care unit, and the assessment of the hip showed stability with $30^{\circ}$ degree of abduction. An abduction orthosis was used to maintain joint reduction, but 4 days later, a further recurrent dislocation was diagnosed (Fig. 5). Closed reduction was performed but stability in reduction was not achieved; a trans-articular Steinmann pin, across the greater trochanter into the acetabulum, with additional supracondylar skeletal traction was used to maintain reduction (Fig. 6). The use of the Steinmann pin instead of an external fixator was a compromise to avoid transfer of the seriously ill patient to the operating room. The patient recovered well subsequently from his multiple injuries and was discharged to the orthopaedic inpatient

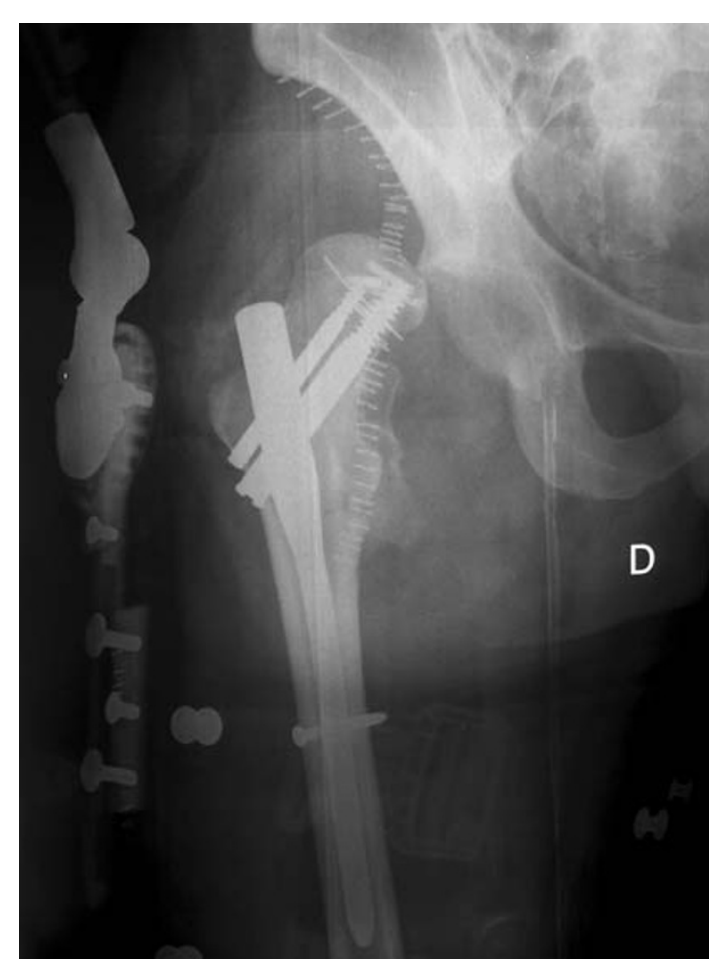

Fig. 5 Recurrent posterior dislocation of the hip despite use of an abduction orthosis 


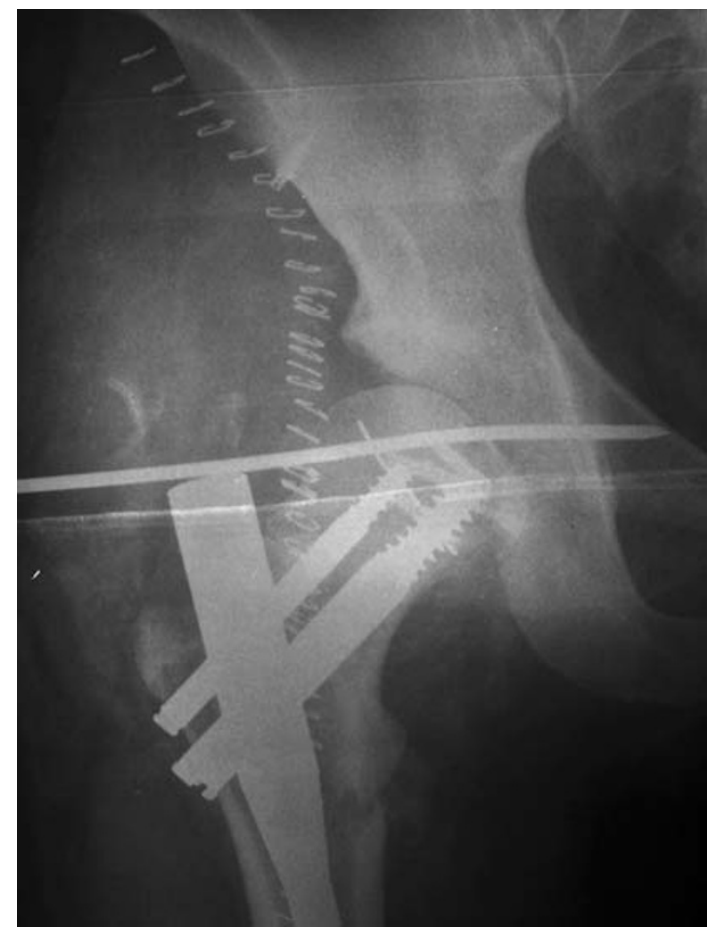

Fig. 6 Steinmann pin across greater trochanter into the acetabulum and supracondylar skeletal traction were used to maintain reduction

unit. One month later, the Steinmann pin was removed and the hip found to remain stable. Following recovery from his neurological, thoracic and abdominal injuries, the patient was allowed up non-weight bearing with crutches and supported in a hip abduction orthosis. At 12 weeks, 25\% weight-bearing was permitted and the hip orthosis discontinued. Radiographs at this stage showed early heterotopic ossification (Fig. 7). He was allowed 50\% weight-bearing at 4 months and advanced to weight-bearing as tolerated at 7 months. Passive range of motion of the hip was assessed and recorded as $60^{\circ}$ flexion, full extension and $20^{\circ}$ abduction. At 18-months, the fractures were healed (Fig. 8). The patient used one crutch and had $60^{\circ}$ flexion, no pain and a Harris Hip Score of 79.1 points. The patient was satisfied with the result and declined further procedures to remove heterotopic ossification.

Patient permission was obtained prior to the publication of this paper.

\section{Discussion}

Fracture-dislocations of the hip are challenging to treat. Dislocation of the hip associated with a proximal femoral fracture is a rare injury, with diagnostic and therapeutic difficulties and potential for a poor outcome [1, 2, 5, 9, 10]. Most studies are limited by a small number of

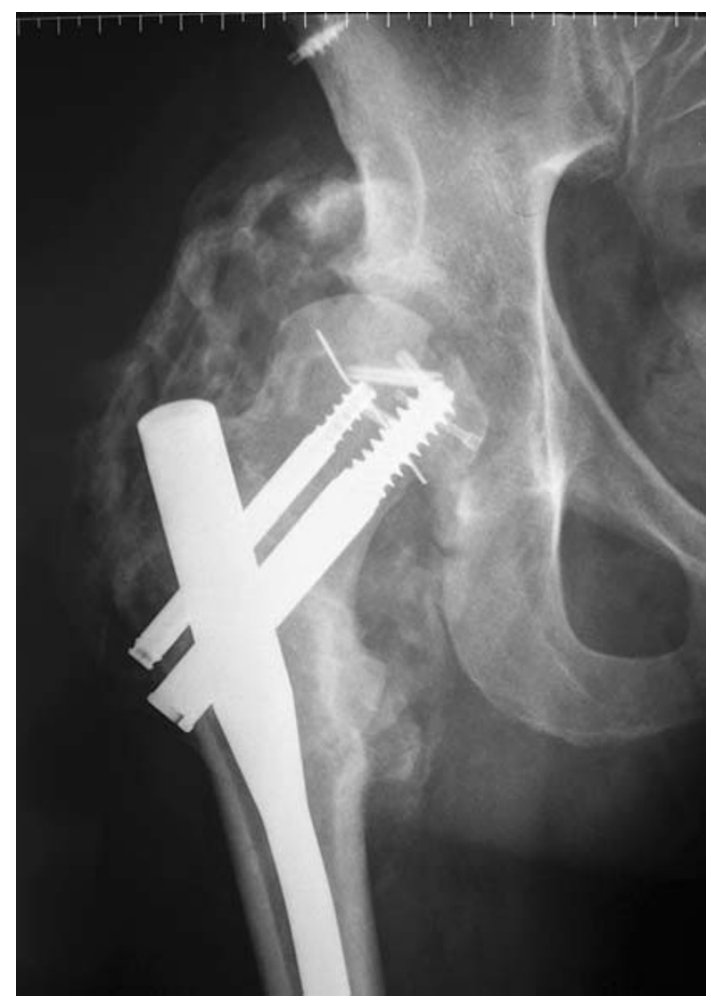

Fig. 7 Three-months follow-up. X-ray showing heterotopic ossification without avascular necrosis of the femoral head

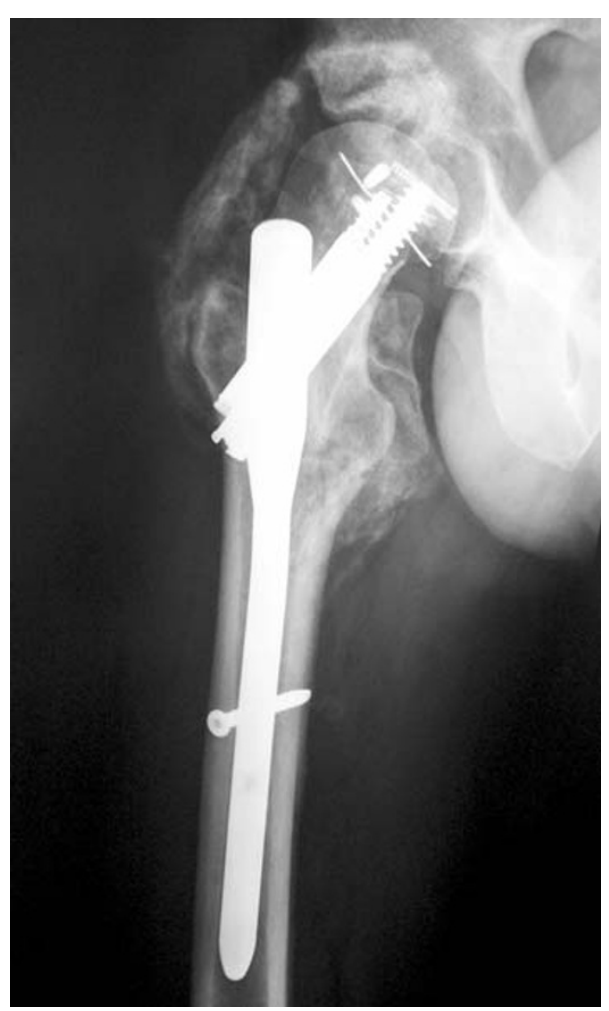

Fig. 8 Final radiograph shows healed fractures and heterotopic ossification 
patients, a wide variety of treatments and relatively shortterm follow-ups [2].

These injuries are the result of high-energy mechanisms with associated multiple organ damage as in this case $[1,2$, $5,9]$. The high-energy transfer during impact is not dissipated with the femoral head fracture alone but propagates further, causing the intertrochanteric fracture [5] and greater vascular damage [11]. Accurate diagnosis is essential [9] and a clear anteroposterior view of the pelvis, including both hips, and a lateral of the injured hip are necessary. This could be supplemented by oblique views and a CT scan $[4,7,9]$. Immediate reduction is mandatory unless the condition of the patient does not allow it. Based on the outcome data of Marchetti et al. [10], the definitive surgical procedure to the hip and femur can be delayed to allow stabilisation of the patient's condition, as in this case. Open reduction has become the primary treatment for hip dislocations associated with femoral head fractures. The management of these lesions can include fragment excision, fixation using countersunk AO screws, Herbert screws and bioabsorbable pins $[2,6]$, or even arthroplasty $[5,7]$. There are different surgical approaches that can be used: anterior, lateral or posterior [8]; many authors prefer the anterior approach because it permits less estimated blood loss, shorter surgical times and better visualisation and fixation. However, it has the theoretical disadvantage of producing greater vascular damage and the development of more clinically significant heterotopic ossification [3]. In contrast, Marchetti et al. [10] did not observe a statistically significant difference between these different approaches. A standard rehabilitation protocol for this type of injury has not been reported but this patient, like others reported, began weight-bearing at $25 \%$ from 3 months, $50 \%$ weightbearing at 4-5 months and to an 'as-tolerated' level at 6-7 months. Although it is anticipated that these patients will have a poor prognosis, the outcome thus far has been reasonable. This may be due to the short-term follow-up that is found with previous and this current report.

The main complications associated with this type of injury are avascular necrosis of the femoral head, heterotopic ossification, peripheral nerve damage and osteoarthritis $[1,3,5,8,9]$; this patient had Brooker type III heterotopic ossification that produced some functional limitation; radiotherapy and indomethacin have both been shown to be of benefit for prophylaxis against heterotopic ossification but was not possible owing to the general status of the patient at the time.

In conclusion, we report a rare variant of a fracturedislocation of the hip where anatomical reduction and fixation were important for achieving an acceptable outcome, even if performed at a delayed stage. This patient had a Harris Hip score of 79.1, with no evidence of AVN at 18-months follow-up, and was satisfied with the result.

Acknowledgments Neither of the authors received payments or other benefits or a commitment or agreement to provide such benefits from a commercial entity in support of their research for or preparation of this article. No commercial entity paid or directed, or agreed to pay or direct, any benefits to any research fund, foundation, educational institution, or other charitable or not profit organisation with which the authors are affiliated or associated.

\section{References}

1. Barquet A, Mussio A (1983) Fracture dislocation of the femoral head with associated ipsilateral trochanteric and shaft fracture of the femur. Arch Orthop Trauma Surg 102:61-63

2. Stannard JP, Harris HW, Volgas DA, Alonso JE (2000) Functional outcome of patients with femoral head fractures associated with hip dislocations. Clin Orthop Relat Res 377:44-56 (Review)

3. Henle P, Kloen P, Siebenrock KA (2007) Femoral head injuries: Which treatment strategy can be recommended? Injury 38(4): 478-488

4. Roeder LF Jr, DeLee JC (1980) Femoral head fractures associated with posterior hip dislocation. Clin Orthop Relat Res 147:121-130

5. Khan M, Wright V, Prayson M (2007) Ipsilateral Intertrochanteric and Pipkin Fractures: An Unusual Case. Am J Orthop 36(4):E53-E55

6. Hougaard K, Thomsen PB (1988) Traumatic posterior fracturedislocation of the hip with fracture of the femoral head or neck, or both. J Bone Joint Surg Am 70(2):233-239

7. Lang-Stevenson A, Getty CJ (1987) The Pipkin fracture-dislocation of the hip. Injury 18(4):264-269 (Review)

8. Epstein HC (1974) Posterior fracture-dislocations of the hip; long-term follow-up. J Bone Joint Surg Am 56(6):1103-1127

9. Mostafa MM (2001) Femoral head fractures. Int Orthop 25(1):51-54

10. Marchetti ME, Steinberg GG, Coumas JM (1996) Intermediateterm experience of Pipkin fracture-dislocations of the hip. J Orthop Trauma 10(7):455-461

11. Bartonícek J, Fric V, Skála-Rosenbaum J, Dousa P (2007) Avascular necrosis of the femoral head in pertrochanteric fractures: a report of 8 cases and a review of the literature. J Orthop Trauma 21(4):229-236 